obachtung und Erfahrung beruhen, zeigt es sich einmal mehr, daß Beobachtung und Erfahrung in der Wissenschaft und in der Praxis unbegrenzten Bestand haben, während den theoretischen Ansichten häufig nur ein ephemeres Leben beschieden ist.

G. Senn.

\title{
Literatur
}

1936 Scheu, Georg, Mein Winzerbuch, Reichsnährstand-Verlags-G. m. b. H. Berlin N. 4.

1910 Schmitthenner, F., Weinbau und Weinbereitung. Aus Natur und Geisteswelt, 332. Bändchen, B. G. Teubner, Leipzig.

J933 Senn, G., Die Entwicklung der biologischen Forschungsmethode in der Antike und ihre grundsätzliche Förderung durch Theophrast von Eresos. VIII. Band der Veröffentlichungen d. Schweiz. Ges. f. Geschichte d. Medizin u. d. Naturwissenschaften, H. R. Sauerländer \& Co., Aarau u. Leipzig.

1912 Struck, Adolf, Zur Landeskunde von Griechenland, Kulturgeschichtliches und Wirtschaftliches. IV. Serie, 4. Heft der «Angewandten Geographie». H. Keller, Frankfurt a. M.

\section{Einiges aus der Praxis von Galen}

Wenn man sich mit den Ärzten der Antike beschäftigt, so kann man diese Aufgabe von einem doppelten Standpunkt aus in Angriff nehmen. Entweder forscht man ihren medizinischen Gedankengängen nach, untersucht, was für neue Hypothesen sie aufgestellt, auf wessen Fußstapfen sie sich weiterentwickelt haben und dergleichen mehr. Oder man beschränkt sich lediglich darauf, sich nur ein anschauliches Bild von ihrer medizinischen Tätigkeit zu machen, m. a. W. sie mehr persönlich zu erfassen, um sich dadurch eine Vorstellung von ihrer ärztlichen Tätigkeit zu verschaffen. Diese letztere Betrachtungweise soll in dieser Arbeit bezüglich der Gestalt Galen's die wegleitende sein.

Gebürtiger Kleinasiate - wahrscheinlich geboren im Jahre 129 n. Chr. - hatte er nach mehrjährigem medizinischem Studium in Smyrna, Korinth und Alexandria als 28jähriger Arzt sich in Pergamon niedergelassen, wo er von dem amtierenden Oberpriester zum alleinigen Gladiatorenarzt ernannt wurde. Interessant ist es, $\mathrm{da} ß$ er gerade die ältesten Schulen, speziell Alexandria, für seine Bildung aufsuchte, wo im 4. und 3. vorchristlichen Jahrhundert unter Herophilos und Erasistratos ein neues medizinisches Leben aufblühte, das sich mit einer Umstellung der bisherigen anatomischen und physiologischen Probleme beschäftigte. Vor einigen Jahren ist es gelungen, die Stätte von Galen's damaliger Tätigkeit, 
das Asklepieion zu Pergamon, genau zu bestimmen, wo er seine Gladiatoren behandelte.

Seine medizinische Tätigkeit lag damals vor allem auf dem Gebiete der Verletzungen, wozu selbstredend auch die Behandlung interner Krankheiten hinzu trat. Auffallend ist es, daß er schon nach relativ kurzer Zeit, d. h. nach 4 Jahren, sich entschloß, seine bisherige Tätigkeit aufzugeben und nach Rom überzusiedeln, das damals als die geistige Metropole Europas galt.

Was ihn zu diesem Entschlusse bewog, wissen wir leider nicht; wir können aber wohl ahnen, daß ehrgeizige Bestrebungen mit im Spiele waren, die ihn zu diesem Schritt veranlaßten. Denn man muß nicht vergessen, daß eine gewisse Selbstüberhebung und ein gewisser Hochmut Zeit seines Lebens ihm eigen waren. Widerspruch konnte er überhaupt nicht vertragen. In diesem letzteren Punkte war er, wie er selbst bekennt, von seiner Mutter aus erblich belastet gewesen. So war es nur allzu begreiflich, daß sein Verhältnis zu den römischen Kollegen gleich von Anfang an kein allzu gutes war. Kaum war er einige Zeit in Rom gewesen, als er beschloß, bei seinen Krankenbesuchen nicht mehr als das Allernotwendigste zu sprechen, und nicht mehr öffentlich als Redner aufzutreten. An seinen Werken nur sollten sie ihn erkennen, nicht aus seinen Worten. Wir wissen aus seinen eigenen Schriften, daß er das große Auditorium im Friedenstempel des Kaisers Vespasian mehrfach zu seinen öfentlichen Vorträgen benutzt hatte.

Doch gehen wir von diesen kurzen persönlichen Bemerkungen zu seiner ärztlichen Tätigkeit über, aus der ich nur einige Punkte herausgreifen möchte.

Nach Rom übergesiedelt, hatte er in den ersten Wochen seiner Praxis das große Glück, einen sogenannten Renommierfall behandeln zu können, der in den weitesten Kreisen rasch bekannt wurde. Es handelte sich dabei um die Erkrankung eines kleinasiatischen Landmannes namens Eudemos, der an Malaria erkrankt war, und zwar am Quartanfieber. Galen konnte genau das Wiederauftreten und Verschwinden des Fiebers voraussagen, was in der Umgebung seines Patienten einen großen Eindruck hervorrief. Man darf nicht unerwähnt lassen, wenn man von seiner römischen ärztlichen Tätigkeit spricht, daß er sich hinsichtlich seines medizinischen Spezialgebietes wesentlich umstellte. Hatte er in Kleinasien hauptsächlich Chirurgie und Orthopädie betrieben, so überließ er Fälle, die in dieses Gebiet einschlugen, nunmehr den römischen Chirurgen und 
wandte sich mehr internen Patienten zu, wobei er auch, wie aus seinen erhaltenen Schriften deutlich hervorgeht, hin und wieder augenärztliche Fälle behandelte oder sich mit Kranken gerne beschäftigte, die ins Gebiet der Neurologie gehörten. Gynäkologie scheint er weniger getrieben zu haben. Als eine große Seltenheit für damalige Zeiten mag ein ophthalmologischer Fall Erwähnung finden, den er - weil klinischer Behandlung sehr bedürftig - in sein Haus aufnahm und ihn hier unter genauer Pflege ausheilte. Es hatte sich dabei um einen phlegmonösen Prozeß an dem einen Auge gehandelt.

Haben wir hier Anfänge einer exakten klinischen Behandlung vor uns, so mutet es uns umso eigenartiger an, wenn Galen gerade in seinen späteren Jahren augenärztliche Patienten auf Distanz behandelte, indem er ihnen auf schriftlichem Wege Mitteilung über die anzuwendenden Mittel zukommen ließ, ohne sie selbst gesehen zu haben. Hatte er doch sonst auf genaue symptomatische Untersuchung seiner Kranken großes Gewicht gelegt. Die Tatsache, daß er einen Kranken in sein eigenes Haus zur Behandlung aufnahm, erscheint uns für unser heutiges Gefühl als etwas ganz Selbstverständliches; für damalige Begriffe muß es als etwas Außergewöhnliches betrachtet werden; denn die Einführung von öffentlichen Krankenhäusern bezw. einer geschlossenen klinischen Behandlung ist für das Abendland erst als eine Errungenschaft des Christentums zu betrachten und taucht zirka im 4 . Jahrhundert auf.

Interessant ist ferner die Erwähnung eines Falles von einem jungen Mann, der sich mit einem Schreibgriffel die Pupille verletzte:

«Sofort floß der wässerige Humor heraus; dann wurde die Pupille kleiner und die ganze Cornea erschien runzeliger. Aber schließlich genas der Knabe und fand sein richtiges Sehvermögen wieder; denn der Humor, der ausgeflossen war, sammelte sich allmählich wieder. Doch ist so etwas sehr selten; meist folgt nämlich derartigen Verletzungen Blindheit.»

Es hatte sich in diesem Fall, den Galen als einen ganz außergewöhnlichen hinstellt, um eine kleine traumatische Perforation der Hornhaut gehandelt mit nachfolgendem Abfluß des Kammerwassers. Die Vorderkammer stellte sich aber rasch wieder her, sodaß das Sehvermögen keine Einbuße erlitt, da zweifellos keine Verletzung der Linse erfolgt war. Galen weiß in diesem Gebiet genau Bescheid, wenn er hinzufügt, daß solche Verletzungen, die gewöhnlich mit ausgedehnter Geschwürsbildung der Horn- 
haut und einer Starentwicklung verbunden sind, häufig zur Erblindung führen.

Noch interessanter ist wohl ein anderer Augenfall, der einen Jüngling betraf, welcher an einem tiefgehenden Hornhautgeschwür erkrankt war und an heftigen Schmerzen litt. Der behandelnde Augenarzt hatte dagegen einen Aderlaß vorgenommen und eine anästhesierende Salbe verordnet, die aus Bleiweiß, Amylum und Mohn bestand. Galen, der in diesem Fall als Consiliarius zugezogen wurde, war mit der angewandten Therapie nicht einverstanden, indem er statt der schmerzlindernden Salbe warme Umschläge verordnete, die mit einem in warmes Wasser getauchten Schwamm vorgenommen wurden. Als der Patient Galen erwiderte, er habe dieses Mittel schon von sich aus versucht, ohne allerdings eine wesentliche Erleichterung zu verspüren, da verordnete Galen ein Vollbad, das ihm die Schmerzen vollkommen benahm, sodaß er die ganze Nacht hindurch schlafen konnte. Durch mehrmalige Vornahme dieser Prozedur wurde der Kranke völlig von seinen Schmerzen geheilt.

Ein Punkt seiner ärztlichen Tätigkeit, in dem er für die damalige Zeit wirklich Großes leistete, muß unbedingt näher beleuchtet werden: ich meine seine psychotherapeutische Behandlungsweise. Hierin hat er die hippokratische Schule um ein Wesentliches überholt. Die Hippokratiker faßten bekanntlich die psychischen Krankheiten auf als bedingt durch Störungen der Viersäftemischungen und richteten die Behandlung infolgedessen darnach ein, indem sie mit medikamentösen Mitteln gegen dieselben vorgingen. Galen dagegen hatte, angeregt durch die Lektüre der römischen und griechischen Philosophen, die Anschauung vertreten, daß geistige Krankheiten zumeist psychisch bedingt seien und nichts mit körperlichen Störungen zu tun hätten. Infolgedessen suchte er solche Fälle durch Suggestion und Psychotherapie zu heilen und hatte dabei auffallende Erfolge zu verzeichnen. Da Galen selbst zu jenen Naturen gehörte, welche ein feines Verständnis und Einfühlungsvermögen für die Krankheiten der menschlichen Psyche hatten, so ist es begreiflich, daß er auf diesem Gebiet sich gerne betätigte und über manche Heilerfolge berichten konnte, welche ihm neidische Kollegen arg mißgönnten. Mehrere Fälle von geheilter Hysterie und Neurasthenie wirkten auf das Publikum äußerst eindrucksvoll und erhöhten sein ärztliches Ansehen in kurzer Zeit, etwa so wie wir dasselbe zirka 1700 Jahre später bei Cagliostro wiederum erleben.

Dem raschen Aufstieg seiner ärztlichen Tätigkeit in Rom (162-166) 
folgt ein auffallend rascher Abbruch derselben, indem er mehr oder weniger fluchtartig diese Stadt verläßt. Können wir uns die Motive einigermaßen vorstellen, welche ihn seinerzeit zum ersten Lokalwechsel von Pergamon nach Rom veranlaßt hatten, so ist es hier wesentlich schwieriger, die eigentlichen Gründe zu erkennen. Zugegeben muß ja werden, daß sein Verhältnis zu manchen der damaligen römischen Ärzte kein allzu gutes war. $\mathrm{Da}$ a aber dieses Moment allein einen solch fluchtartigen Ortswechsel bedingt haben sollte, ist kaum anzunehmen. Man wird wohl kaum mehr die eigentlichen Gründe dieser Flucht aus Rom näher feststellen können. Sehr unwahrscheinlich erscheint es mir, daß man von mehrfacher Seite aus das Auftreten der Pest im Jahre 166 als ursächliches Motiv hinstellen wollte. Man kann es sich kaum vorstellen, daß ein Arzt von der Größe eines Galen sich vor einer herannahenden Epidemie sollte gefürchtet haben.

Doch dieser selbstgewählte Unterbruch seiner römischen Tätigkeit sollte durch eine höhere Schicksalsfügung nach einigen Jahren wieder rückgängig gemacht werden. 4 Jahre nach seinem Weggang wird er vom römischen Kaiser Mark Aurel aufgefordert, nach Rom zurückzukehren. Er läßt sich von ihm dazu bestimmen und tritt nunmehr seine zweite römische Tätigkeit an, die zirka 30 Jahre dauern sollte. Durch diese Wiederberufung nach Rom war er zum offiziellen Leibarzt des Kaisers gestempelt worden. Will man sich heutzutage ein oberflächliches Bild von der Tätigkeit eines fürstlichen Leibarztes machen, so geht man wohl nicht fehl, wenn man annimmt, daß seine Tätigkeit hauptsächlich darin liegt, bei eventuellen leichten oder schwereren Indispositionen: wie Grippe, Erkältungskrankheiten und ähnlichem, die erste Behandlung einzuleiten, wozu natürlich in jüngster Zeit noch gelegentliche Sport- und Verkehrsunfälle hinzukommen mögen. Für einen damaligen kaiserlichen Leibarzt erstreckte sich die ärztliche Tätigkeit auf die Behandlung von wesentlich anderen Krankheiten.

In erster Linie waren es schwere Magen- und Darmstörungen, Folge der lukullischen Lebensweise, welche damals die Fürstenhöfe in hohem Grade kennzeichnete, sodaß es wohl nicht als ein reiner Zufall angesehen werden darf, daß Galen gerade zum ersten Male, als er zum Kaiser gerufen wurde, eine schwere Darmerkrankung bei ihm konstatierte, die er durch strenge diätetische Maßnahmen zur großen Zufriedenheit seines hohen Herrn ausheilte.

Magen- und Darmstörungen der verschiedensten Art wurden damals 
nicht mit einer Schonungsdiät wie heutzutage, d.h. mit Schleimsuppen und püreartigen Speisen behandelt, sondern man suchte im Gegenteil durch eine Reiztherapie das erkrankte Organ wieder zur normalen Funktion anzuregen. So verordnete Galen in solchen Fällen häufig starken Rotwein, dem Pfeffer und andere reizende Gewürze beigegeben waren.

Galen spricht sich an mehreren Stellen offen darüber aus, daß der Luxus in den höheren Gesellschaftsklassen soweit gestiegen sei, daß an eine Überbietung kaum mehr gedacht werden könne. Daneben mußte ein Hofmedicus der damaligen Zeit gut Bescheid wissen auf dem Gebiete der Gifte und Gegengifte; denn der Giftmord an hohen Persönlichkeiten war in jener Zeit an der Tagesordnung. So mußten die damaligen Ärzte, die gleichzeitig auch Apotheker waren, genaue Kenntnisse nicht bloß von Giften, sondern auch von entsprechenden Gegengiften besitzen. Endlich muß noch einer dritten Erkrankung Erwähnung getan werden, die im damaligen Rom bei Arm und Reich eine große Rolle spielte. Ich meine die Malaria in ihren verschiedenen Erscheinungsformen. Da Amerika damals noch nicht entdeckt und infolgedessen die Chinarinde noch völlig unbekannt war, machte die Behandlung dieser Krankheit große Schwierigkeiten, so daß sie erst nach längerer Zeit zur Ausheilung gebracht werden konnte. Die damaligen Ärzte behalfen sich hauptsächlich mit Aderlässen sowie diätetischen und hydrotherapeutischen Maßnahmen. Da eine Temperaturmessung mittels Instrumenten noch nicht vorgenommen werden konnte und außerdem die Perkussion und Auskultation noch unbekannt waren, so verlegte man sich vor allem darauf, die verschiedenen Qualitäten des Pulses möglichst genau zu prüfen und zu registrieren, um daraus weitere Schlüsse zu ziehen. Darin war Galen ein Meister, wie er überhaupt die verschiedenen Symptome einer Krankheit möglichst genau zu eruieren suchte. Die Beobachtung, daß die Malaria in einer Tertiana- und Quartanaform auftritt, aus welcher er das Wiederauftreten des Fiebers mit großer Genauigkeit voraussagen konnte, hat Galen wohl darauf gebracht, auch bei anderen Krankheiten auf die sogen. kritischen Tage zu achten - ein Bestreben, das in unseren Tagen wieder von neuem Boden gewonnen hat, wenngleich zugegeben werden muß, daß aus solchen Beobachtungen lange nicht die weitreichenden Folgerungen gezogen werden können, wie man es ursprünglich glaubte.

Es zeugt von großer Gewissenhaftigkeit, wenn Galen bei manchen 
Krankheitsfällen wie beispielsweise bei komplizierten Knochenbrüchen, mitunter die ganze Nacht am Krankenbette des Betreffenden zubrachte, da ihm die Gegenwart eines Assistenten nicht zuverlässig genug erschien. Ebenso gewissenhaft verfährt er bei einem Sklaven, der 7 Tage lang das Faulfieber hatte, und den er regelmäßig bei Tag und bei Nacht besuchte. Ob einer reich oder arm war, das spielte bei der Behandlung seiner Patienten nicht die geringste Rolle.

Neben den erwähnten Krankheiten, die damals häufig zur Behandlung kamen, wäre noch die gefährliche Elephantiasis zu nennen, die in den meisten Fällen durch die bekannte Filaria hominis bedingt ist, einem Parasiten, der aus den römischen Kolonien vielfach nach Rom eingeschleppt wurde. Über die Behandlungsmethoden dieser Krankheit ist leider nichts Näheres angegeben.

Als besonders bemerkenswert sei erwähnt, daß Galen bereits davon Kenntnis hatte, daß eine Krankheit durch Überimpfung einer andern geheilt werden könne, und zwar handelte es sich um die Übertragung der Lepra auf einen Elephanthiasiskranken, der dadurch geheilt wurde. $\mathrm{Ob}$ es sich hiebei, wie Herzog vermutet, um eine reine Lepra gehandelt hat, möchte ich bezweifeln, da dieses griechische Wort außer Aussatz auch eine schuppende Hautkrankheit bezeichnet, welch letztere Interpretation wohl die wahrscheinlichere sein dürfte. Es ist äußerst interessant, daß der Gedanke einer Krankheitsübertragung zu Heilzwecken nach zirka 1800 Jaren von neuem wieder aufgenommen wurde für die Heilung bestimmter infektiöser Nervenleiden insbesondere der Paralyse.

Eine Frage ist wohl noch wert, kurz aufgerollt zu werden. Ich meine die Frage der Antiseptica bei infizierten Wunden. Chemische Antiseptica in unserem modernen Sinne gab es damals noch nicht. Man war also auf «Naturstoffe» angewiesen. Als einen solchen hat Galen in Anlehnung an medizinische Vorläufer den herben Rotwein empfohlen in der richtigen Erkenntnis, daß Alkohol und Gerbsäure wirksame Stoffe gegen Infektionen darstellen. Diese Methode wurde noch von hervorragenden Chirurgen im Mittelalter geübt, so z. B. von Mondeville in Montpellier um 1304.

Im Anschluß an die Anwendung des Rotweins bei infizierten Wunden sei noch kurz eines vielgebrauchten antiken Allheilmittels gedacht, das fast 2000 Jahre lang regelmäßig Anwendung fand und noch um das Jahr 1800 in der französischen Pharmakopoe figurierte. Es ist dies der vielgenannte Theriak. Dieses Heilmittel wurde schon in der vorgalenischen Zeit im Altertum, namentlich aber im späteren Altertum und im Mit- 
telalter als eine reine Panacee gegen die verschiedensten Krankheiten gefeiert. Seinem Wesen nach besteht der Theriak aus einer höchst komplizierten, im einzelnen stark schwankenden Komposition der verschiedensten Ingredienzien, unter welchen das Schlangenfleisch eine besondere Rolle spielte.

Ursprünglich war der Theriak, weil er Extrakte von Schlangenfleisch enthielt, als Heilmittel gegen Schlangenbisse angegeben worden, bekam aber dann im Laufe der Zeit die bekannte stark erweiterte Anwendungsweise. $\mathrm{Da} \beta$ aber gegenüber solchen irrationalen Vorstellungen der gesunde Menschenverstand damals schon in manchen Fällen das Richtige traf, geht aus einer Bemerkung Galen's hervor, die uns besagt, daß ein Winzer, der von einer Schlange gebissen war, sich selbständig dadurch rettete, daß er sich sofort mit einem Taschenmesser den Finger abschnitt.

Zur weiteren Illustrierung der ärztlichen Tätigkeit Galens mag ein Faktum dienen, das uns heutzutage wohl etwas eigenartig anmutet. Zahlreiche Patienten, hauptsächlich aus den besten Kreisen der damaligen Gesellschaft, suchten ihn in seiner Sprechstunde auf, um sich kosmetisch von ihm beraten zu lassen, und zwar in bezug auf Haarausfall, Haarfärbung, Vermeidung von Runzeln und dergleichen mehr, ein Metier, das heutzutage ein Arzt vom Range Galens neidlos einem Coiffeur oder Verschönerungskünstler überlassen würde. Wir müssen aber dabei bedenken, daß die Haar- und Hautpflege damals bei den höhern Klassen eine größere Rolle spielte wie heutzutage. Wurde es doch im alten Rom für eine vornehme Frau als unpassend angesehen, mit grauen Haaren herumzugehen. Lassen Sie mich nur zwei diesbezügliche therapeutische Punkte herausgreifen:

Als ein ausgezeichnetes. Mittel gegen Haarausfall erwähnt Galen das Nardenöl, ein indisches Mittel, das uns bereits im Talmud überliefert ist. Auch Mittel, die er aus der «Kosmetik» der Königin Kleopatra übernommen hatte, empfiehlt er angelegentlich seinen Patienten. Alternden Frauen, die ihre Haare färben wollen, rät er an, als unschädliches Mittel das Cedernharz zu verwenden. Es kann aber dieses Mittel kaum anders als ein Bindemittel aufgefaßt werden, dem irgendwelche färberische Stoffe beigegeben waren, über die allerdings keine näheren Angaben vorliegen.

Daß Galen nicht nur das medizinisch überlieferte Wissen seinen Schülern weitergegeben hat, sondern selbst von einem hohen Forschungsgeist beseelt war, dafür spricht sein Tadel, welchen er einigen Militär- 
ärzten zukommen ließ, weil sie die Gelegenheit unbenützt vorübergehen ließen, im Germanenkrieg die Leichen der gefallenen Feinde zu sezieren. Sie hätten dabei viel mehr lernen können als durch die Sektionen von Tieren. Er selbst hatte bekanntlich nur in einigen Fällen - wahrscheinlich waren es hingerichtete Verbrecher - Gelegenheit gehabt, eine Sektion an menschlichen Leichen vorzunehmen. Leider aber hat seine große Eitelkeit und Selbsteingenommenheit diesem Forschungsstreben manchmal einen starken Dämpfer aufgesetzt. So ermahnt er seine Schüler, wenn sie es später einmal zur Berühmtheit bringen wollten, nur das in sich aufzunehmen, was er in eifriger Forschung während seines Lebens festgestellt habe, und sich nicht durch andere Lehren beeinflussen zu lassen.

Es ist sehr zu bedauern, daß seine Nachfolger sich allzu sehr an diesen Appell gehalten haben, sodaß in der Folgezeit die medizinische Wissenschaft wiederum in ein Stadium der Stagnation geriet und sich für lange nicht mehr weiter entfalten konnte. E. Wölfflin.

\section{Schweizerische Bibliographie der Geschidhte der Naturwissenschaften und der Medizin. 1938-1943, II. Serie}

1. Geschichte der Naturwissenschaften: Allgemeines, Bibliographie.

Caizzi, Bruno. Alcune note sulla classificazione delle scienze e sulla filosofia biologica di Agostino Cournot. Boll. Soc. ticin. sci. nat., anno 33, 1938, p. $122-128$.

Fisenring, Max E. Johann Heinrich Lambert und die wissenschaftliche Philosophie der Gegenwart. Promotionsarbeit Math. Techn. Hochschule Zürich VIII + 113 S. $21 \times 15 \mathrm{~cm}$. Zürich Buchdr. Müller, Werder \& Co. AG., 1942.

Heuß, Eugen. Rationale Biologie und ihre Kritik. (Eine Auseinandersetzung mit dem Vitalismus H. Driesch's). Diss. phil. I. Univ. Zürich. zugleich: Studien u. Bibliogr. z. Gegenwartsphil., H. 24, 1938, 193 S.

Kopp, Josef Vitalis. Das physikalische Weltbild der frühen griechischen Dichtung. Ein Beitrag zum Verständnis der vorsokratischen Physik. Diss. Phil. Freiburg, Schweiz. VIII + 333 S. Freiburg, Schweiz. Paulusdruckerei, 1939.

Misteli, Hermann. Carl Vogt. Seine Entwicklung vom angehenden naturwissenschaftlichen Materialisten zum idealen Politiker der Paulskirche (1817-1849). Diss. phil. I Bern. zugl.: Schweiz. Studien z. Geschichtswiss. Bd. 19, 1938, geol. pal. Teil. S. 22-48. 